\title{
Deduction of pure spin current from the linear and circular spin photogalvanic effect in semiconductor quantum wells
}

\author{
Bin Zhou ${ }^{1,2}$ and Shun-Qing Shen ${ }^{1}$ \\ ${ }^{1}$ Department of Physics and Center for Theoretical and Computational Physics, The University of Hong Kong, \\ Pokfulam Road, Hong Kong, China \\ ${ }^{2}$ Department of Physics, Hubei University, Wuhan 430062, China
}

(Received 18 October 2006; published 25 January 2007)

\begin{abstract}
We study the spin photogalvanic effect in a two-dimensional electron system with structure-inversion asymmetry by means of solution of the semiconductor optical Bloch equations. It is shown that linearly polarized light may inject a pure spin current into spin-splitting conduction bands due to Rashba spin-orbit coupling, while circularly polarized light may inject a spin-dependent photocurrent. We establish an explicit relation between the photocurrent caused by oblique incidence of circularly polarized light and the pure spin current caused by normal incidence of linearly polarized light such that we can deduce the amplitude of the spin current from the experimentally measured spin photocurrent. This method may provide a source of spin current to study spin transport in semiconductors quantitatively.
\end{abstract}

DOI: 10.1103/PhysRevB.75.045339

PACS number(s): 72.25.Fe, 72.40.+w

\section{INTRODUCTION}

Spin-coherent transport of conduction electrons in semiconductor heterostructures is currently an emerging subject due to its possible application in a new generation of electronic devices. ${ }^{1}$ There have been considerable efforts to achieve spin-polarized current or pure spin current (PSC) in semiconductors. Optical injection of spin current is based largely on the fact that the spin-polarized carriers in conduction band can be injected in semiconductors via absorption of the polarized light. In the case of semiconductors, if the photon energy is higher than a characteristic energy gap, such as that of the conduction and valence bands of electrons, or that between subbands, electrons are pumped into the conduction band from the valence band or conduction subband. When the inversion symmetry of the system is broken, the singlephoton absorption may generate a spin current or a spinpolarized current. The circular photogalvanic effect (CPGE), which is based on converting the helicity of light into an electric current by irradiation with circularly polarized light, has been studied extensively. ${ }^{2-10}$ The conventional CPGE focuses only on the charge, not the spin aspect of electronic transport in semiconductors. It was first realized that quantum interference of one- and two-photon excitation of unbiased semiconductors may yield a ballistic spin-polarized current, which was observed by two groups. ${ }^{11-13}$ Recently it was proposed that one-photon absorption of linearly polarized light should produce a PSC in the noncentrosymmetric semiconductors. ${ }^{14-17}$ On the other hand, experimental detection of the PSC has been realized by measuring spin accumulation near the boundary of samples ${ }^{18,19}$ and the electric current induced by the PSC in a crossbar system. ${ }^{20-22}$

Structure-inversion asymmetry (SIA) in semiconductor heterojunctions may lead to spin splitting of the conduction band in the momentum $\mathbf{k}$ space and induce spin-orbit coupling. This type of system may be a good candidate to implement spin-based electronic devices and has attracted more and more attentions. In this paper, we investigate how to deduce the PSC in semiconductor quantum wells (QWs) by irradiation of linearly and circularly polarized light. By using the solution of the semiconductor optical Bloch equations, we establish an explicit relation between the spin photocurrent induced by oblique incidence of circularly polarized light and the PSC with in-plane spin polarization induced by normal incidence of linearly polarized light with the same frequency and intensity. Since the photocurrent can be measured experimentally, we can deduce the PSC from the measured photocurrent based on several material-specific parameters. This method can provide an efficient source for generating a PSC quantitatively, and has potential applications in semiconductor spintronics.

\section{MODEL AND GENERAL FORMALISM}

We consider a QW of zinc-blende-type semiconductors with SIA. The conduction electrons can be modeled as

$$
H_{c}=\frac{\hbar^{2} k^{2}}{2 m^{*}}-\lambda \hbar\left(k_{x} \sigma_{y}-k_{y} \sigma_{x}\right),
$$

where $\sigma$ are the Pauli matrices, $\lambda$ is the strength of Rashba spin-orbit coupling, and $m^{*}$ is the effective mass of the conduction electron. The valence bands near the $\Gamma$ point are described approximately by the Luttinger Hamiltonian for spin $S=3 / 2$ holes,

$$
H_{L}=-\frac{\hbar^{2}}{2 m}\left[\left(\gamma_{1}+\frac{5}{2} \gamma_{2}\right) k^{2}-2 \gamma_{2}(\mathbf{k} \cdot \mathbf{S})^{2}\right],
$$

where $\gamma_{1}, \gamma_{2}$ are two Kohn-Luttinger parameters, $m$ is the free electron mass, and $\mathbf{S}$ represents three $4 \times 4$ spin-3/2 matrices. For a bulk system, both heavy- and light-hole bands are degenerate at the $\Gamma$ point. In a QW with thickness $L$, while $k_{x}$ and $k_{y}$ are good quantum numbers, the confinement along the $z$ axis is approximately realized by taking $\left\langle k_{z}\right\rangle=0$ and $\left\langle k_{z}^{2}\right\rangle \simeq(\pi / L)^{2}$ for the lowest energy band. In the case of $k^{2}=k_{x}^{2}+k_{y}^{2} \ll\left\langle k_{z}^{2}\right\rangle$, the energy spectrum of the first doubly degenerate heavy-hole band is reduced approxi- 


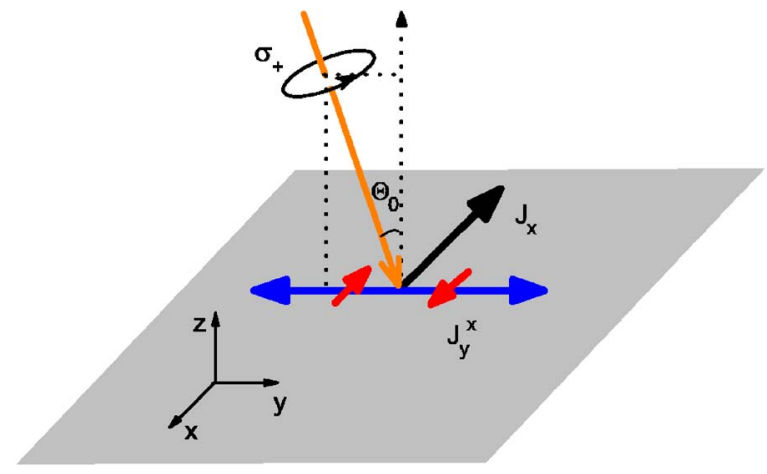

FIG. 1. (Color online) Sketch of right-handed circularly polarized $\left(\sigma_{+}\right)$light irradiating on the surface of a semiconductor QW in the $(y z)$ plane with incidence angle $\Theta_{0}$. In this case, the photocurrent $J_{x}$ is injected perpendicularly to the incident plane of the light, and the PSC $J_{y}^{x}$ is also injected. Orange arrow denotes the direction of light propagation; thick black arrow denotes photocurrent; blue arrows denote PSC with in-plane spin polarization represented by red arrows.

mately to $E^{H H} \simeq-\hbar^{2} k^{2} /\left(2 m_{H H}\right)-\varepsilon$, with the effective mass $m_{H H}=m /\left(\gamma_{1}+\gamma_{2}\right)$ and $\varepsilon=\hbar^{2}\left\langle k_{z}^{2}\right\rangle\left(\gamma_{1}-2 \gamma_{2}\right) /(2 m)$. Finite thickness of the QW makes the band structure into a sequence of quasi-two-dimensional (2D) subbands with $\left\langle k_{z}^{2}\right\rangle \simeq(n \pi / L)^{2}(n$ is a nonzero integer), which can be calculated numerically. ${ }^{23}$ Of course, for the precise calculations, we need to take into account the band structure of the whole $\mathbf{k}$ space. In the present paper, we first consider this simplified 2D model and then present numerical results by taking into account the finite thickness effect on the band structure near the $\Gamma$ point.

Now we come to study a system irradiated with polarized light with incidence angle $\Theta_{0}$ in the plane $(y z)$ as shown in Fig. 1. The pump pulse is of the form

$$
\mathbf{E}(t)=\mathbf{E}_{\omega} e^{-i\left(\omega t-k \cos \Theta_{0} z+k \sin \Theta_{0} y\right)}+\text { c.c. },
$$

where $\omega$ is the frequency of the light. By treating the field perturbatively, and assuming fast interband dephasing, the semiconductor optical Bloch equations give the singleparticle density matrix in conduction bands due to optical irradiation ${ }^{14,24-26}$

$$
\begin{aligned}
\rho_{c c^{\prime}}(\mathbf{k})= & \frac{\pi e^{2}}{\hbar^{2}} \sum_{v} \frac{\mathbf{E}_{\omega} \cdot \mathbf{v}_{c v}}{\omega_{c v}} \frac{\mathbf{E}_{\omega}^{*} \cdot \mathbf{v}_{v c^{\prime}}}{\omega_{c^{\prime} v}} \\
& \times\left[\delta\left(\omega-\omega_{c v}\right)+\delta\left(\omega-\omega_{c^{\prime} v}\right)\right] \tau_{e},
\end{aligned}
$$

where the subscripts $c$ and $v$ refer to conduction and valence bands, $\mathbf{v}_{c v}(\mathbf{k})=\langle c \mathbf{k}|\mathbf{v}| v \mathbf{k}\rangle$ is the interband matrix element of the velocity operator, $\tau_{e}$ is the momentum relaxation time as a result of all the various interactions, and $\hbar \omega_{c\left(c^{\prime}\right) v}$ $=\hbar^{2} k^{2} /(2 \mu) \pm \lambda \hbar k+\Delta_{0}$ [with $\Delta_{0}$ being the band gap and the reduced mass $\left.\mu=m^{*} m_{H H} /\left(m^{*}+m_{H H}\right)\right]$ for the simplified 2D model. Using this solution, a physical observable $\mathbf{O}$ in the conduction bands can be calculated by

$$
\mathbf{O}=\sum_{c, c^{\prime}, \mathbf{k}}\left\langle c^{\prime} \mathbf{k}|\hat{O}| c \mathbf{k}\right\rangle \rho_{c c^{\prime}}(\mathbf{k})
$$

where $\hat{O}$ is the corresponding operator. In the following, the spin current operator $\hat{J}_{i}^{j}$ is defined conventionally as $\hat{J}_{i}^{j}$ $=(\hbar / 4)\left\{v_{i}, \sigma_{j}\right\}$.

\section{SPIN CIRCULAR PHOTOGALVANIC EFFECT}

Spin photocurrent in the CPGE has been studied extensively. Here we focus on the spin aspect of the CPGE. Consider oblique incidence of circularly polarized light onto the system. In this case a spin photocurrent can circulate perpendicular to the incident plane of the light. When the light enters the sample, due to the refraction effect, the light can be described by $E_{x}=E_{0} t_{s} \cos \varphi, E_{y}=i E_{0} t_{p} \sin \varphi \cos \Theta$, and $E_{z}=i E_{0} t_{p} \sin \varphi \sin \Theta$, where $E_{0}$ is the electric field amplitude in vacuum, $\Theta$ is the angle of refraction defined by $\sin \Theta$ $=\sin \Theta_{0} / n \quad(n$ is the index of refraction $), t_{s}$ $=2 \cos \Theta_{0} /\left(\cos \Theta_{0}+n \cos \Theta\right)$ and $t_{p}=2 \cos \Theta_{0} /\left(n \cos \Theta_{0}\right.$ $+\cos \Theta)$ are transmission coefficients after Fresnel's formula for linear $s$ and $p$ polarization. ${ }^{27}$ The helicity of the incident light is $P_{\text {circ }}=\left(I_{\sigma_{+}}-I_{\sigma_{-}}\right) /\left(I_{\sigma_{+}}+I_{\sigma_{-}}\right)=\sin 2 \varphi$, where $I_{\sigma_{+}}$and $I_{\sigma_{-}}$ are the intensities of right- $\left(\sigma_{+}\right)^{-}$and left-handed $\left(\sigma_{-}^{+}\right)$polarized radiation. $P_{\text {circ }}= \pm 1$ denotes right and left circularly polarized light, respectively. In this way the photocurrent can be calculated explicitly. ${ }^{26}$ The hole current induced in the valence bands is neglected because the effective mass of holes is typically much greater than that of electrons, and the kinetic energy and speed of holes are much less than those of electrons. ${ }^{28}$ In the oblique incidence of circularly polarized light in the (yz) plane, the formula (5) gives the photocurrent $J_{y}=0$ and

$$
J_{x}=-\frac{2 \lambda \mu^{3} \Omega}{3 \hbar^{5}\left\langle k_{z}^{2}\right\rangle m^{*}} t_{s} t_{p} a_{0}^{2} e^{3} E_{0}^{2} \tau_{e} P_{\text {circ }} \sin \Theta,
$$

where $\Omega=\lambda^{2} \mu+\hbar \omega-\Delta_{0}$ and $a_{0}=\sqrt{6}\langle 0,0|x| 1,-1\rangle$, which is a parameter determined by experiment. It is clear that the photocurrent circulates only in the case of circularly polarized light $\left(P_{\text {circ }} \neq 0\right)$, and vanishes in the case of linearly polarized light $\left(P_{\text {circ }}=0\right)$. In addition to the photocurrent in CPGE, a PSC with $x$ component of spin polarization perpendicular to the direction of the photocurrent also circulates,

$$
J_{y}^{x}=\left(I_{+}^{C} t_{p}^{2} \cos ^{2} \Theta \sin ^{2} \varphi+I_{-}^{C} t_{s}^{2} \cos ^{2} \varphi\right) \hbar a_{0}^{2} e^{2} E_{0}^{2} \tau_{e},
$$

where

$$
I_{ \pm}^{C}=\frac{\lambda \mu}{6 \hbar^{5}\left\langle k_{z}^{2}\right\rangle m^{*}}\left[\hbar^{2}\left\langle k_{z}^{2}\right\rangle\left(m^{*}-\mu\right) \pm \mu^{2} \Omega\right] .
$$

The spin current even survives in normal incidence while the photocurrent vanishes. Figure 1 shows that the photocurrent $J_{x}$ and PSC $J_{y}^{x}$ are induced by right-handed circularly polarized light irradiating on the surface of a semiconductor QW in the $(y z)$ plane with incidence angle $\Theta_{0}$.

Absorption of circularly polarized light in semiconductors induces a $z$ component of spin polarization $S^{z}$ due to the conservation of angular momentum. The light-induced non- 

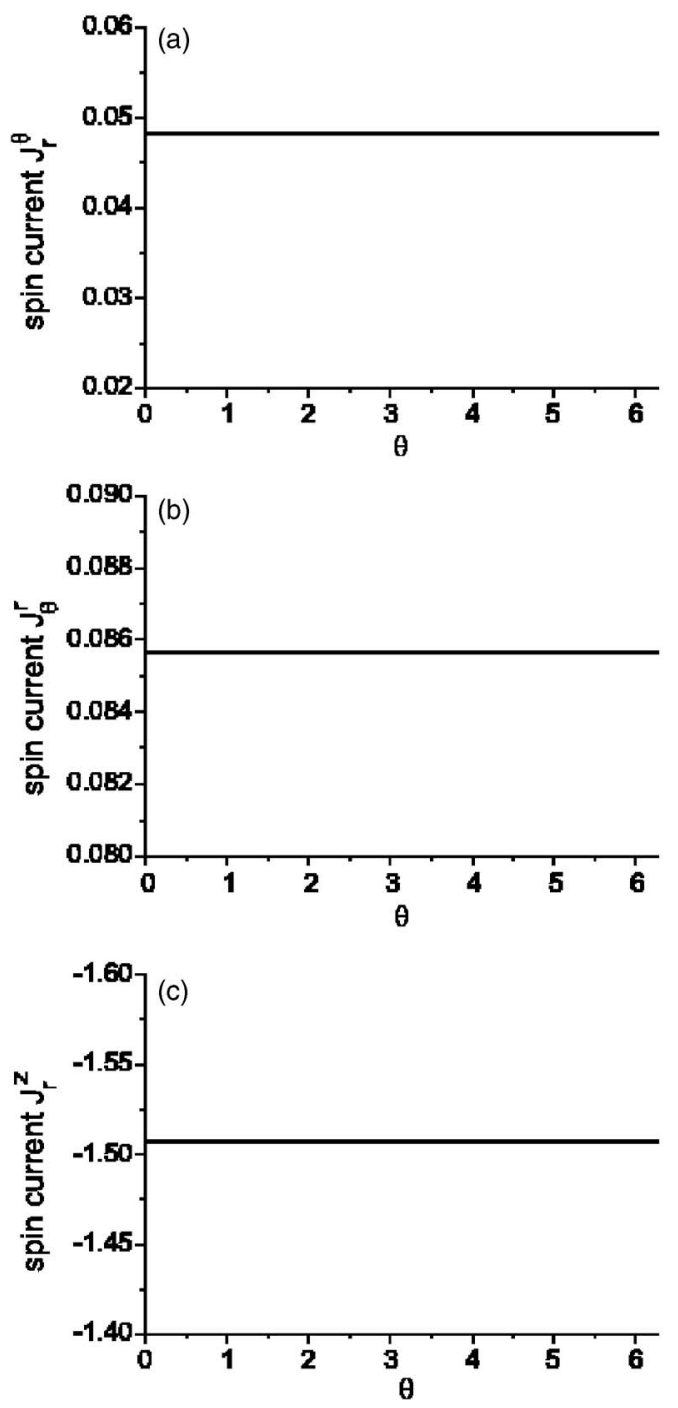

FIG. 2. (Color online) Orientational distribution of pure spin current in the case of normally incident $\sigma_{+}$-polarized light. (a)-(c) Numerical calculation results (in units of $10^{-3} \mathrm{eV}^{-2} \mathrm{~nm}^{-1} \mathrm{fs}^{-2}$ $\left.\times \hbar a_{0}^{2} e^{2} E_{0}^{2} \tau_{e}\right)$. The experiment parameters are taken as $\Delta_{0}$ $=750 \mathrm{meV}, \quad \lambda=6.3 \mathrm{meV} \mathrm{nm} / \hbar$, $m^{*}=0.05 m, \quad \gamma_{1}=6.9, \quad \gamma_{2}=2.2, \quad L$ $=14 \mathrm{~nm}$, the index of refraction $n=\sqrt{13}$, and the wavelength of the light $\lambda_{0}=1620 \mathrm{~nm}$. (d)-(f) Sketches of orientational distribution, in which red arrows (light gray) denote the directions of spin current, blue arrows (dark gray) denote the polarization directions of spin current, and $\otimes$ denotes spin polarization along $-z$ direction. (a) and (d) for $J_{r}^{\theta}(\theta)$; (b) and (e) for $J_{\theta}^{r}(\theta)$; (c) and (f) for $J_{r}^{z}(\theta)$.

zero $S^{z}$ will lead to an orientational distribution of PSCs with the $z$-component polarization

$$
J_{r}^{z}(\theta)=-\frac{\mu^{2} \Omega}{6 \hbar^{3} m^{*} \pi \delta} t_{s} t_{p} \hbar a_{0}^{2} e^{2} E_{0}^{2} \tau_{e} P_{\text {circ }} \cos \Theta
$$

with $\theta=\arctan \left(k_{x} / k_{y}\right), \delta=\sqrt{\mu\left(\lambda^{2} \mu+2 \hbar \omega-2 \Delta_{0}\right)}$, and the subscript $r$ denoting the radial direction in polar coordinates. However, one has $J_{x(y)}^{z}(\mathbf{k})=J_{x(y)}^{z}(-\mathbf{k})$ such that the total spin current with $z$-component polarization vanishes. With the geometric constraint of the sample, a PSC with $z$ component of polarization can circulate and may be used to implement the reciprocal spin Hall effect. ${ }^{29}$ In the case of normally incident $\sigma^{+}$-polarized light, the orientational distributions of radial spin current with tangent direction polarization [i.e., $\left.J_{r}^{\theta}(\theta)\right]$ and tangent spin current with radial direction polarization [i.e., $\left.J_{\theta}^{r}(\theta)\right]$ are given by

$$
J_{r}^{\theta}(\theta)=\frac{\lambda \mu\left(2 \mu-m^{*}\right)}{12 \hbar^{3} m^{*} \pi} t_{0}^{2} \hbar a_{0}^{2} e^{2} E_{0}^{2} \tau_{e}
$$

$$
J_{\theta}^{r}(\theta)=\frac{\lambda \mu}{12 \hbar^{3} \pi} t_{0}^{2} \hbar a_{0}^{2} e^{2} E_{0}^{2} \tau_{e}
$$

where the sub- and superscript $\theta$ denotes the tangent direction in polar coordinates. The total contribution of the orientational distributions of spin current leads to a nonvanishing spin current with in-plane spin polarization as shown in Eq. (7).

For the sake of clarity, the orientational distributions of spin current in the case of normally incident $\sigma^{+}$-polarized light are plotted in Fig. 2.

\section{SPIN LINEAR PHOTOGALVANIC EFFECT}

Now we come to consider the normal incidence of linearly polarized light onto the sample, and the pump pulse is of the form

$$
\mathbf{E}(t)=\mathbf{E}_{\omega} \exp [-i(\omega t-k z)]+\text { c.c. }
$$

In the medium, $E_{x}=t_{0} E_{0} \cos \phi$ and $E_{y}=t_{0} E_{0} \sin \phi$, with $t_{0}$ $=2 /(1+n)$ and $\phi$ the angle between the polarization plane and the $x$ axis, e.g., $\phi=0$, corresponding to $x$-polarized light. 

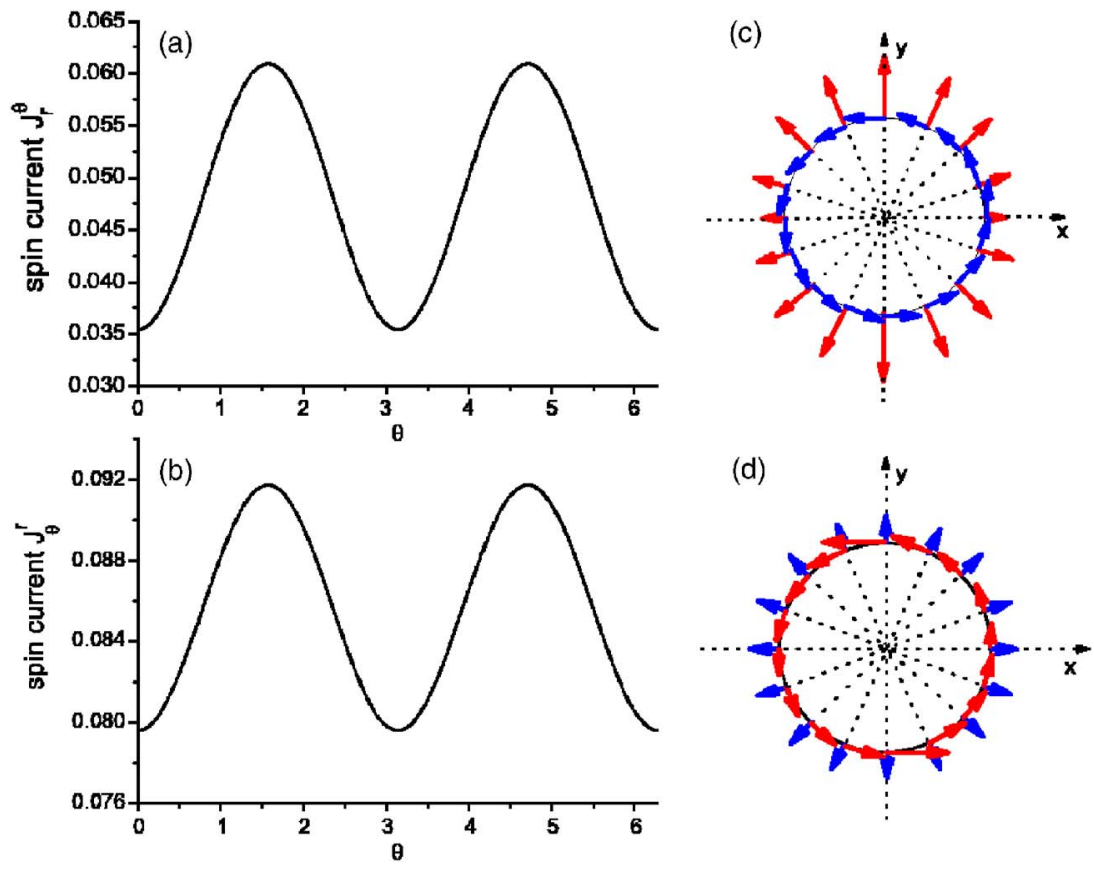

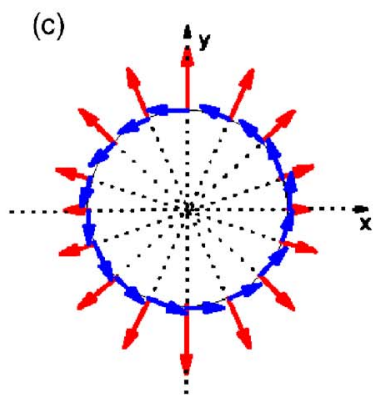

FIG. 3. (Color online) Orientational distribution of pure spin current in the case of normally incident $x$-polarized light. (a),(b) Numerical calculation results (in units of $10^{-3} \mathrm{eV}^{-2} \mathrm{~nm}^{-1} \mathrm{fs}^{-2}$ $\left.\times \hbar a_{0}^{2} e^{2} E_{0}^{2} \tau_{e}\right)$. The experimental parameters are taken as the same as those given in Fig. 2. (c),(d) Sketches of orientational distribution, in which red arrows (light gray) denote the directions of spin current, and blue arrows (dark gray) denote the polarization directions of spin current. (a) and (c) for $J_{r}^{\theta}(\theta)$; (b) and (d) for $J_{\theta}^{r}(\theta)$.
In this case it is known that no photocurrent is injected as in Eq. (6). However, a PSC may survive. The physical origin of the spin current is given briefly as follows. Due to Rashba spin-orbit coupling, the conduction band splits into two subbands denoted by $|\uparrow\rangle$ and $|\downarrow\rangle$. When the frequency $\omega$ of the light satisfies the condition $\hbar \omega>\Delta_{0}$, electrons are pumped from the heavy-hole band into conduction bands. If there appears an electron state $|\mathbf{k}, \uparrow\rangle$ in the conduction band $|\uparrow\rangle$ with momentum $\mathbf{k},|-\mathbf{k}, \downarrow\rangle$ must appear in the conduction band $|\downarrow\rangle$ with momentum $-\mathbf{k}$ with the same probability according to the symmetry. $|\mathbf{k}, \uparrow\rangle$ and $|-\mathbf{k}, \downarrow\rangle$ are two degenerate states and have opposite velocities; thus the pair contributes a null electric current. However, they have opposite spin polarization such that they carry equal spin current. Therefore a finite spin current survives for these two states. The spin splitting of the conduction band plays an essential role in this mechanism. ${ }^{21,22}$ As an example, the orientational distributions of spin current in the case of normally incident $x$-polarized light are plotted in Fig. 3.

An explicit calculation gives a PSC with in-plane spin polarization,

$$
\begin{gathered}
J_{x}^{y}=\left(-I_{0}^{L}-I_{1}^{L} \cos 2 \phi\right) t_{0}^{2} \hbar a_{0}^{2} e^{2} E_{0}^{2} \tau_{e}, \\
J_{x}^{x}=I_{1}^{L} t_{0}^{2} \hbar a_{0}^{2} e^{2} E_{0}^{2} \tau_{e} \sin 2 \phi,
\end{gathered}
$$

where $I_{0}^{L}=\lambda \mu\left(m^{*}-\mu\right) /\left(6 \hbar^{3} m^{*}\right)$ and $I_{1}^{L}=\lambda \mu^{3} \Omega /\left(6 \hbar^{5} m^{*}\left\langle k_{z}^{2}\right\rangle\right)$. It is obvious that a PSC with in-plane spin polarization is dependent on the angle $\phi$ between the polarization plane and the $x$ axis. Linearly polarized light can be decomposed as a combination of two circularly polarized beams of light. The phase difference between these two composite beams of the light is $2 \phi$. The polarization dependence of the PSC originates from the interference of two composite circularly polarized beams of light.

\section{RELATION BETWEEN PHOTOCURRENT AND SPIN CURRENT}

The two formulas for photocurrent in Eq. (6) and spin current in Eq. (13) contain the parameter $a_{0}$ and the relaxation time $\tau_{e}$, which need to be determined experimentally. Assuming the intensity and the frequency of the two applied light beams are equal, the ratio of the photocurrent of circularly polarized light incident with an oblique angle $\Theta_{0}$ to the spin current of normally incident linear polarized light gives

$$
\frac{J_{x}}{J_{x}^{y}}=\eta \frac{t_{s} t_{p}}{t_{0}^{2}} P_{\text {circ }} \sin \Theta \frac{2 e}{\hbar},
$$

where $\eta$ is a dimensionless frequency-dependent factor,

$$
\eta=\frac{2 \Omega}{\epsilon_{0}+\Omega \cos 2 \phi},
$$

with $\epsilon_{0}=\hbar^{2}\left\langle k_{z}^{2}\right\rangle\left(m^{*}-\mu\right) / \mu^{2}$. For a small incidence angle $\Theta_{0}$ and $P_{\text {circ }}=1$, the ratio is reduced to $J_{x} / J_{x}^{y} \approx \eta\left(\Theta_{0} / n\right) 2 e / \hbar$. In this way we establish an explicit relation between lightinjected photocurrent and PSC. All parameters in $\eta$ are known in semiconductor materials. For the sample of InGaAs, ${ }^{10}$ for instance, $\Delta_{0}=750 \mathrm{meV}, \lambda=6.3 \mathrm{meV} \mathrm{nm} / \hbar$, $m^{*}=0.05 m, \gamma_{1}=6.9, \gamma_{2}=2.2, L=14 \mathrm{~nm}, \epsilon_{0}=50.8 \mathrm{meV}$, and $\Omega=\hbar \omega-749.982 \mathrm{meV}$. In this system, the photocurrent $J_{x}$ in the CPGE has been measured successfully. Therefore we can deduce the spin current by measuring the photocurrent experimentally.

\section{NUMERICAL RESULTS}

The formula in Eq. (16) is valid only for excitation of electrons near the $\Gamma$ point. In principle we can calculate the ratio of photocurrent to spin current following the $\mathbf{k} \cdot \mathbf{p}$ calculation done by Baht et al. ${ }^{14}$ Here we present our results 


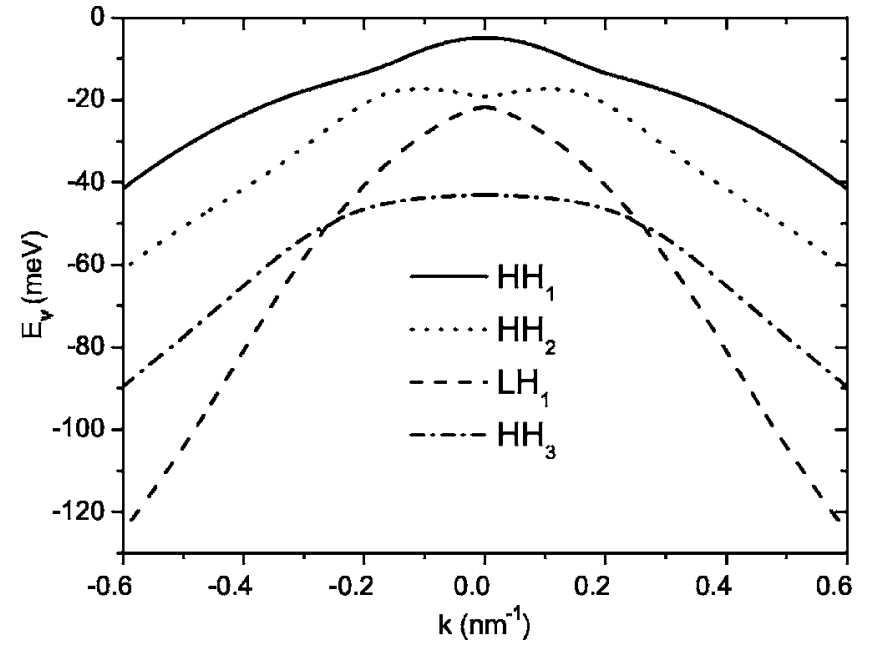

FIG. 4. Dispersion of low-lying subbands for the Luttinger effective Hamiltonian with the width $L=14 \mathrm{~nm}, \gamma_{1}=6.9$, and $\gamma_{2}$ $=2.2$. HH and LH denote heavy hole and light hole, respectively.

after the quantum size effect of a QW with a finite thickness $L$ is taken into account. For a confining potential $V(z)$ along the $z$ axis, say $V(z)=+\infty$ for $|z|>L / 2$ and $V(z)=0$ for otherwise. While $k_{x}$ and $k_{y}$ remain as good quantum numbers, the quantization along the $z$ axis can be calculated numerically by the truncation approximation if $L$ is of order of tens of nanometers. ${ }^{22,30}$ The lowest four valence subbands are plotted in Fig. 4, where each subband is doubly degenerate.

In this way the photocurrent and PSC can be calculated numerically in terms of the unknown parameters $a_{0}$ and $\tau_{e}$. The variation of $J_{x}$ in the case of obliquely incident $\sigma_{+}$-polarized light with the frequency of light is plotted in Fig. 5(a), and the photocurrent has its sign change when the dominant contribution of the interband transition to the conduction band switches from the first heavy-hole subband to the second heavy-hole subband. We also plot the spin current $J_{x}^{y}$ in a normally incident $x$-polarized light in Fig. 5(b). The frequency dependence of the dimensionless factor $\eta$ is plotted in Fig. 5(c). When $\hbar \omega$ is close to the band gap $\Delta_{0}$ the main contribution results from the interband transition from the first heavy-hole subband to the conduction band, the ratio factor is linear in the frequency $\omega$. The photocurrent was observed experimentally in two samples of InGaAs with Rashba coupling $\lambda_{1}=3.0 \mathrm{meV} \mathrm{nm} / \hbar$ and $\lambda_{2}$ $=6.3 \mathrm{meV} \mathrm{nm} / \hbar .{ }^{10}$ The photocurrent changes its sign when the frequency of laser increases. In the experiment of Ref.10, the angle dependence of photocurrent is measured experimentally $J_{x}\left(\Theta_{0}\right) \simeq 351 \Theta_{0} / n \mathrm{nA}$ for a small angle $\Theta_{0}$ (with the index of refraction $n=\sqrt{13}$ ). The ratio factor is estimated as $\eta \simeq 0.62$. If we keep our conditions for the laser except that the helicity of light changes from circular to linear; the PSC in linearly polarized light is estimated as $J_{x}^{y}$ $\simeq 566 \hbar / 2 e \mathrm{nA}$.

\section{CONCLUSION}

Here we use a model with a twofold conduction band described by Rashba coupling and a valence band described
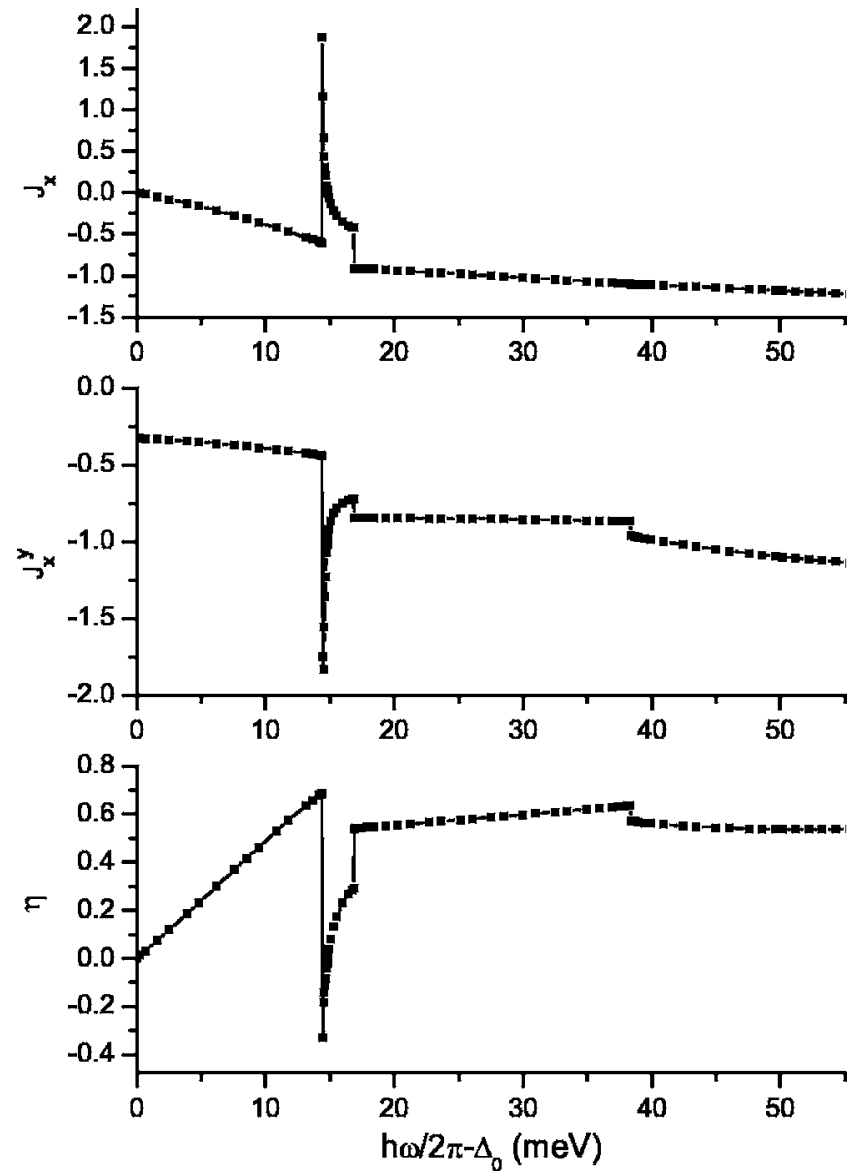

FIG. 5. Numerical results based on a model of a QW with a finite thickness $L$. The parameters are given in the text following Eq. (12). (a) Spectrum of photocurrent $J_{x}$ in the case of obliquely incident $\sigma_{+}$-polarized light (in units of $10^{-3} \mathrm{eV}^{-2} \mathrm{~nm}^{-1} \mathrm{fs}^{-2}$ $\times t_{s} t_{p} a_{0}^{2} e^{3} E_{0}^{2} \tau_{e} \sin \Theta$ ); (b) spectrum of spin current $J_{x}^{y}$ in normally incident $x$-polarized light (in units of $10^{-3} \mathrm{eV}^{-2} \mathrm{~nm}^{-1} \mathrm{fs}^{-2}$ $\times t_{0}^{2} \hbar a_{0}^{2} e^{2} E_{0}^{2} \tau_{e}$ ); (c) frequency dependence of the dimensionless factor $\eta(\omega)$.

by the Luttinger Hamiltonian to investigate the spin photogalvanic effect induced by polarized light via interband excitations in the semiconductor QWs. We established a relation between the light-injected photocurrent and the PSC. As the photocurrent in the CPGE was extensively studied both theoretically and experimentally, we can make use of it to deduce the PSC in the same system by using linearly polarized light to replace the circularly polarized light in the CPGE, which can be realized by adding a 1/4-wave plate. Thus this method may provide a source of spin current to study spin transport in semiconductors quantitatively.

\section{ACKNOWLEDGMENTS}

This work was supported by the Research Grant Council of Hong Kong under Grants No. HKU 7039/05P and No. HKU 7042/06P, and the CRCG of the University of Hong Kong. 
${ }^{1}$ Semiconductor Spintronics and Quantum Computation, edited by D. D. Awschalom, D. Loss, and N. Samarth (Springer-Verlag, Berlin, 2002).

${ }^{2}$ E. L. Ivchenko and G. E. Pikus, Pis'ma Zh. Eksp. Teor. Fiz. 27, 640 (1978) [JETP Lett. 27, 604 (1978)].

${ }^{3}$ V. I. Belinicher, Phys. Lett. 66A, 213 (1978).

${ }^{4}$ N. S. Averkiev and M. I. D'yakonov, Fiz. Tekh. Poluprovodn. (S.-Peterburg) 17, 629 (1983) [Sov. Phys. Semicond. 17, 393 (1983)].

${ }^{5}$ D. Hägele, M. Oestreich, W. W. Rühle, N. Nestle, and K. Eberl, Appl. Phys. Lett. 73, 1580 (1998).

${ }^{6}$ J. M. Kikkawa and D. D. Awschalom, Nature (London) 397, 139 (1999).

${ }^{7}$ S. D. Ganichev, H. Ketterl, W. Prettl, E. L. Ivchenko, and L. E. Vorobjev, Appl. Phys. Lett. 77, 3146 (2000).

${ }^{8}$ S. D. Ganichev, E. L. Ivchenko, S. N. Danilov, J. Eroms, W. Wegscheider, D. Weiss, and W. Prettl, Phys. Rev. Lett. 86, 4358 (2001).

${ }^{9}$ S. D. Ganichev and W. Prettl, J. Phys.: Condens. Matter 15, R935 (2003).

${ }^{10}$ C. L. Yang, H. T. He, L. Ding, L. J. Cui, Y. P. Zeng, J. N. Wang, and W. K. Ge, Phys. Rev. Lett. 96, 186605 (2006).

${ }^{11}$ J. Hübner, W. W. Rühle, M. Klude, D. Hommel, R. D. R. Bhat, J. E. Sipe, and H. M. van Driel, Phys. Rev. Lett. 90, 216601 (2003).

${ }^{12}$ M. J. Stevens, A. L. Smirl, R. D. R. Bhat, A. Najmaie, J. E. Sipe, and H. M. van Driel, Phys. Rev. Lett. 90, 136603 (2003).

${ }^{13}$ A. Najmaie, E. Ya. Sherman, and J. E. Sipe, Phys. Rev. Lett. 95, 056601 (2005).

${ }^{14}$ R. D. R. Bhat, F. Nastos, A. Najmaie, and J. E. Sipe, Phys. Rev. Lett. 94, 096603 (2005).
${ }^{15}$ H. Zhao, X. Pan, A. L. Smirl, R. D. R. Bhat, A. Najmaie, J. E. Sipe, and H. M. van Driel, Phys. Rev. B 72, 201302(R) (2005).

${ }^{16}$ S. A. Tarasenko and E. L. Ivchenko, JETP Lett. 81, 231 (2005).

${ }^{17}$ S. A. Tarasenko and E. L. Ivchenko, cond-mat/0609090 (unpublished).

${ }^{18}$ Y. K. Kato, R. C. Myers, A. C. Gossard, and D. D. Awschalom, Science 306, 1910 (2004).

${ }^{19}$ J. Wunderlich, B. Kaestner, J. Sinova, and T. Jungwirth, Phys. Rev. Lett. 94, 047204 (2005).

${ }^{20}$ S. O. Valenzuela and M. Tinkham, Nature (London) 442, 176 (2006).

${ }^{21}$ X. D. Cui, S. Q. Shen, J. Li, W. K. Ge, and F. C. Zhang, condmat/0608546 (unpublished).

${ }^{22}$ J. Li, X. Dai, S. Q. Shen, and F. C. Zhang, Appl. Phys. Lett. 88, 162105 (2006).

${ }^{23} \mathrm{R}$. Winkler, Spin-Orbit Coupling Effects in Two-Dimensional Electron and Hole Systems (Springer-Verlag, Berlin, 2003).

${ }^{24} \mathrm{H}$. Haug and S. W. Koch, Quantum Theory of the Optical and Electronic Properties of Semiconductors (World Scientific, Singapore, 1993).

${ }^{25}$ U. Rössler, Phys. Status Solidi B 234, 385 (2002).

${ }^{26}$ L. E. Golub, Phys. Rev. B 67, 235320 (2003).

${ }^{27}$ E. L. Ivchenko and G. E. Pikus, Superlattices and Other Heterostructures. Symmetry and Optical Phenomena (Springer, Berlin, 1997).

${ }^{28}$ A. Haché, J. E. Sipe, and H. M. van Driel, IEEE J. Quantum Electron. 34, 1144 (1998).

${ }^{29}$ E. M. Hankiewicz, J. Li, T. Jungwirth, Q. Niu, S. Q. Shen, and J. Sinova, Phys. Rev. B 72, 155305 (2005).

${ }^{30}$ R. Winkler, H. Noh, E. Tutuc, and M. Shayegan, Phys. Rev. B 65, 155303 (2002). 